\title{
Induction of oxidative stress by anticancer drugs in the presence and absence of cells
}

\author{
CHIKAKO YOKOYAMA ${ }^{1,2}$, YUTO SUEYOSHI ${ }^{2}$, MIKA EMA $^{2}$, YUMI MORI $^{2}$, \\ KAZUTO TAKAISHI $^{3}$ and HISASHI HISATOMI ${ }^{2}$
}

\begin{abstract}
${ }^{1}$ Department of Biochemical Engineering, Graduate School of Science and Engineering, Yamagata University, Yonezawa, Yamagata 992-8510; ${ }^{2}$ Laboratory of Cellular and Molecular Biochemistry, Department of Materials and Life Sciences, Seikei University, Musashino, Tokyo 180-8633; ${ }^{3}$ Graduate School of Natural Science and Technology, Okayama University, Okayama, Okayama 700-8530, Japan
\end{abstract}

Received September 21, 2015; Accepted February 13, 2017

DOI: $10.3892 / \mathrm{ol} .2017 .6931$

\begin{abstract}
Reactive oxygen species (ROS) are generated in the cell through multiple mechanisms. Intracellular ROS are rapidly detoxified by various enzymatic and non-enzymatic mechanisms; however, disruption of the oxidant-antioxidant balance causes oxidative stress and elicits cell damage. The oxidative stress induced by chemotherapy is known to cause side effects in patients with cancer. However, few studies have examined whether anticancer drugs induce oxidative stress in cancer cells. Furthermore, the precise mechanism by which anticancer drugs induce the generation of ROS remains unclear. In the present study, to investigate whether anticancer drugs induce oxidative stress, DLD-1 human colorectal cancer cells were treated with 20 different anticancer drugs and then stained with CellROX ${ }^{\circledR}$ ROS detection reagent. Furthermore, an oxygen radical absorbance capacity assay in the presence of copper was performed to estimate the oxidative activities of the anticancer drugs in the absence of cells. The data of the present study using assay methods in the presence and absence of cells suggest that nimustine, actinomycin D, doxorubicin, mitomycin C, mitoxantrone, carmofur, gemcitabine, mercaptopurine, camptothecin, paclitaxel, vinblastine, and vinorelbine are able to induce oxidative stress.
\end{abstract}

\section{Introduction}

Reactive oxygen species (ROS) include oxygen molecules $\left(\mathrm{O}_{2}\right)$, superoxide anion radicals $\left(\mathrm{O}_{2}^{-}\right)$, hydroxyl free radicals $\left(\mathrm{HO}^{-}\right)$

Correspondence to: Dr Chikako Yokoyama, Department of Biochemical Engineering, Graduate School of Science and Engineering, Yamagata University, 4-3-16 Jonan, Yonezawa, Yamagata 992-8510, Japan

E-mail: 4koyama@yz.yamagata-u.ac.jp

Key words: anticancer drug, reactive oxygen species, oxidative stress, CellROX ${ }^{\circledR}$, the oxygen radical absorbance capacity assay in the presence of copper and hydrogen peroxide $\left(\mathrm{H}_{2} \mathrm{O}_{2}\right)$. ROS are generated as a result of single- or multi-electron reductions of oxygen by cellular enzymes or in the mitochondrial respiratory pathway (1-5). Although an increase in the level of intracellular ROS leads to oxidative stress and DNA damage, the effects of ROS are normally balanced by antioxidants, such as reduced glutathione (GSH), ascorbic acid, and ureic acid (6). Disruption of the oxidant-antioxidant balance through alterations to cellular homeostasis or defective repair of ROS-induced damage is involved in the pathogenesis of several diseases (7). In particular, it can be the primary trigger and/or mediator of carcinogenesis by contributing to the initiation of cellular malignancy and the progression of cancer (8-10).

Furthermore, it is known that anticancer drugs induce oxidative stress in patients with cancer being treated with chemotherapy. Elevated levels of oxidants in the circulation have been reported in patients with cancer following administration of epirubicin $(11,12)$. Epirubicin and doxorubicin possess an anthracycline skeleton, and generate ROS that lead to DNA damage and subsequently antitumor activity $(13,14)$. Vinblastine and vinorelbine belong to the class of vinca alkaloids. It has been demonstrated that vinorelbine depletes intracellular GSH and increases intracellular ROS production (15). However, few studies have investigated the association between oxidative stress and the effects of anticancer drugs.

Available methods for measuring oxidative stress inducibility include direct measurement of intracellular ROS, indirect measurement of the resulting damage to biomolecules, including proteins, DNA, RNA and lipids, and the detection of antioxidant levels. In the present study, cell staining with CellROX ${ }^{\circledR}$ Green reagent was performed in order to directly measure the intracellular ROS formation (16-18). CellROX $^{\circledR}$ reagent is a fluorogenic probe used for measuring oxidative stress in cells and exhibits bright-green photostable fluorescence upon oxidation by ROS. The oxygen radical absorbance capacity (ORAC) assay is a method for estimating antioxidant activity (19-23) and is often used to determine antioxidant levels in foods. Previous studies have demonstrated that copper $\left(\mathrm{Cu}^{2+}\right)$ undergoes redox cycling reactions and possesses the ability to produce reactive radicals in normal and cancer cells $(24,25)$. Therefore, in the present study the ORAC 
assay in the presence of $\mathrm{Cu}^{2+}\left[\mathrm{ORAC}\left(\mathrm{Cu}^{2+}\right)\right.$ assay] was used for quantification of the oxidant activity of anticancer drugs. In the ORAC $\left(\mathrm{Cu}^{2+}\right)$ assay, the reduction in the fluorescence intensity of fluorescein was calculated as a measure of the oxidant activity of the anticancer drug. In the current study, the ability of 20 anticancer drugs to induce oxidative stress was measured using the methods described above in vitro and in the absence of cells.

\section{Materials and methods}

Cell culture. The DLD-1 human colorectal cancer cell line was purchased from the Cell Resource Center for Biomedical Research at Tohoku University (Sendai, Japan) and maintained in RPMI media (Thermo Fisher Scientific, Inc., Waltham, MA, USA) supplemented with $10 \%$ heat-inactivated fetal bovine serum (Gibco; Thermo Fisher Scientific, Inc.). DLD-1 cells were maintained under standard culture conditions in a $5 \% \mathrm{CO}_{2}$ incubator at $37^{\circ} \mathrm{C}$ until they reached $80-90 \%$ confluence.

Treatment with anticancer drugs. DLD-1 cells were treated for $24 \mathrm{~h}$ in a $5 \% \mathrm{CO}_{2}$ incubator at $37^{\circ} \mathrm{C}$ with $10 \mu \mathrm{M}$ of the following anticancer drugs: Cyclophosphamide, dacarbazine, nimustine, temozolomide, actinomycin $\mathrm{D}$, doxorubicin, mitomycin $\mathrm{C}$, mitoxantrone, carmofur, cytarabine, fluorouracil, gemcitabine, mercaptopurine, carboplatin, oxaliplatin, camptothecin, etoposide, paclitaxel, vinblastine and vinorelbine. Positive control cells were treated with $10 \mu \mathrm{M}$ 2,2'-azobis (2-amidinopropane) dihydrochloride (AAPH), which is a free radical generator. All anticancer drugs and AAPH were obtained from Wako Pure Chemical Industries, Ltd. (Osaka, Japan).

Cell staining with CellROX $X^{\circledR}$ ROS detection reagent. Following incubation with the anticancer drugs or AAPH for $24 \mathrm{~h}, 5 \mu \mathrm{M}$ CellROX $^{\circledR}$ Green reagent (Thermo Fisher Scientific, Inc.) was added to DLD-1 cells and incubated for $30 \mathrm{~min}$ in a $5 \% \mathrm{CO}_{2}$ incubator at $37^{\circ} \mathrm{C}$. The cells were washed with PBS, fixed with $3.7 \%$ formaldehyde in PBS for 15 min and permeabilized with $0.5 \%$ Triton X-100 in PBS for 5 min, at room temperature. Glass coverslips were mounted using SlowFade ${ }^{\circledR}$ Diamond Antifade mountant (Thermo Fisher Scientific, Inc.). The samples were examined using a Leica DM IL LED fluorescence microscope (Leica Microsystems GmbH, Wetzlar, Germany). Cells treated with each anticancer drug were examined at three or more fields of view, and the ratings of -, none or weak; + , slight; ++ , moderate and +++ , severe were manually assigned to the stained cell slides.

ORAC and ORAC $\left(\mathrm{Cu}^{2+}\right)$ assay. Application of the ORAC and ORAC $\left(\mathrm{Cu}^{2+}\right)$ assay determined the fluorescence intensity in the presence of the drug relative to that in the presence of dimethyl sulfoxide (DMSO; Wako Pure Chemical Industries, Ltd.). The ORAC assays contained $50 \mu \mathrm{M}$ of an anticancer drug or $5 \mathrm{mM}$ AAPH, with $10 \mathrm{mM}$ fluorescein (Wako Pure Chemical Industries, Ltd.). The ORAC $\left(\mathrm{Cu}^{2+}\right)$ assay additionally contained $10 \mu \mathrm{M} \mathrm{CuSO}_{4}$ (Wako Pure Chemical Industries, Ltd.). The assay mixtures were incubated for $1.5 \mathrm{~h}$ at room temperature, in the dark. The fluorescence intensity was subsequently measured using a Flex station 3 fluorescence plate reader (Molecular Devices, LLC, Sunnyvale, CA, USA) with filters for excitation wavelength of $494 \mathrm{~nm}$ and emission wavelength of $523 \mathrm{~nm}$. The result was described as the percentage of fluorescence intensity relative to DMSO treatment. The ORAC and ORAC $\left(\mathrm{Cu}^{2+}\right)$ assay was tested once.

Cell staining with Annexin V-Cy3 apoptosis detection reagent. Following incubation with the anticancer drugs for $24 \mathrm{~h}$, Annexin V-Cy3 reagent (dilution, 1:200; BioVision, Inc., Milpitas, CA, USA) was added to the DLD-1 cells and incubated for $5 \mathrm{~min}$ at room temperature, in the dark. The cells were examined using the previously described Leica DM IL LED fluorescence microscope.

Calculation of radical structural energy of anticancer drugs. To calculate the radical structural energy of the previously described anticancer drugs, modeling with the B3LYP/6-31G(d) method was performed in Gaussian version 09 D.01 (Gaussian Inc., Wallingford, CT, USA). The radical structural energies were calculated as $\Delta(\mathrm{E}+\mathrm{ZPE})$ or $\Delta \mathrm{E}(26)$.

\section{Results}

Detection of ROS in DLD-1 cells with CellROX ${ }^{\circledR}$ reagent. The investigated anticancer drugs are classified as follows: i) Alkylating agents-cyclophosphamide, dacarbazine, nimustine and temozolomide; ii) antibiotics-actinomycin $\mathrm{D}$, doxorubicin, mitomycin $\mathrm{C}$ and mitoxantrone; iii) antineoplastic agents-carmofur, cytarabine, fluorouracil, gemcitabine and mercaptopurine; iv) platinating agents-carboplatin and oxaliplatin; and v) vinca alkaloids-camptothecin, etoposide, paclitaxel, vinblastine and vinorelbine. To investigate whether these anticancer drugs induce intracellular ROS formation, DLD-1 cells treated with each anticancer drug were stained with CellROX ${ }^{\circledR}$ Green reagent. As demonstrated in Fig. 1, intracellular ROS formation was induced in DLD-1 cells treated with actinomycin $\mathrm{D}$, carmofur and vinorelbine. DLD-1 cells treated with vinorelbine exhibited the strongest CellROX $^{\circledR}$ Green signal among all anticancer drugs. The results of CellROX ${ }^{\circledR}$ staining are summarized in Table I. Furthermore, certain anticancer drugs, including vinblastine and vinorelbine, markedly decreased the number of cells. It was initially assumed that the anticancer drugs had induced growth inhibition or apoptotic cell death. Annexin V-Cy3 staining indicated that the cells treated with actinomycin D, doxorubicin, mitomycin $\mathrm{C}$, vinblastine or vinorelbine had undergone apoptotic cell death (data not shown).

Quantification of oxidant activity using the ORAC $\left(\mathrm{Cu}^{2+}\right)$ assay. The ORAC and ORAC $\left(\mathrm{Cu}^{2+}\right)$ assays are methods for measuring the antioxidant ability of food. In the present study, the ORAC $\left(\mathrm{Cu}^{2+}\right)$ assay was used to determine the oxidant activities of anticancer drugs in the absence of cells by measuring the reduction of fluorescence intensity derived from fluorescein. As demonstrated in Table I, the abilities of nimustine, mitoxantrone, gemcitabine and vinblastine to reduce the fluorescence intensity were comparable to that of AAPH, whereas vinorelbine markedly reduced the fluorescence intensity. These results suggested that nimustine, mitoxantrone, gemcitabine, vinblastine and vinorelbine may directly generate intracellular ROS. In addition, the ORAC 

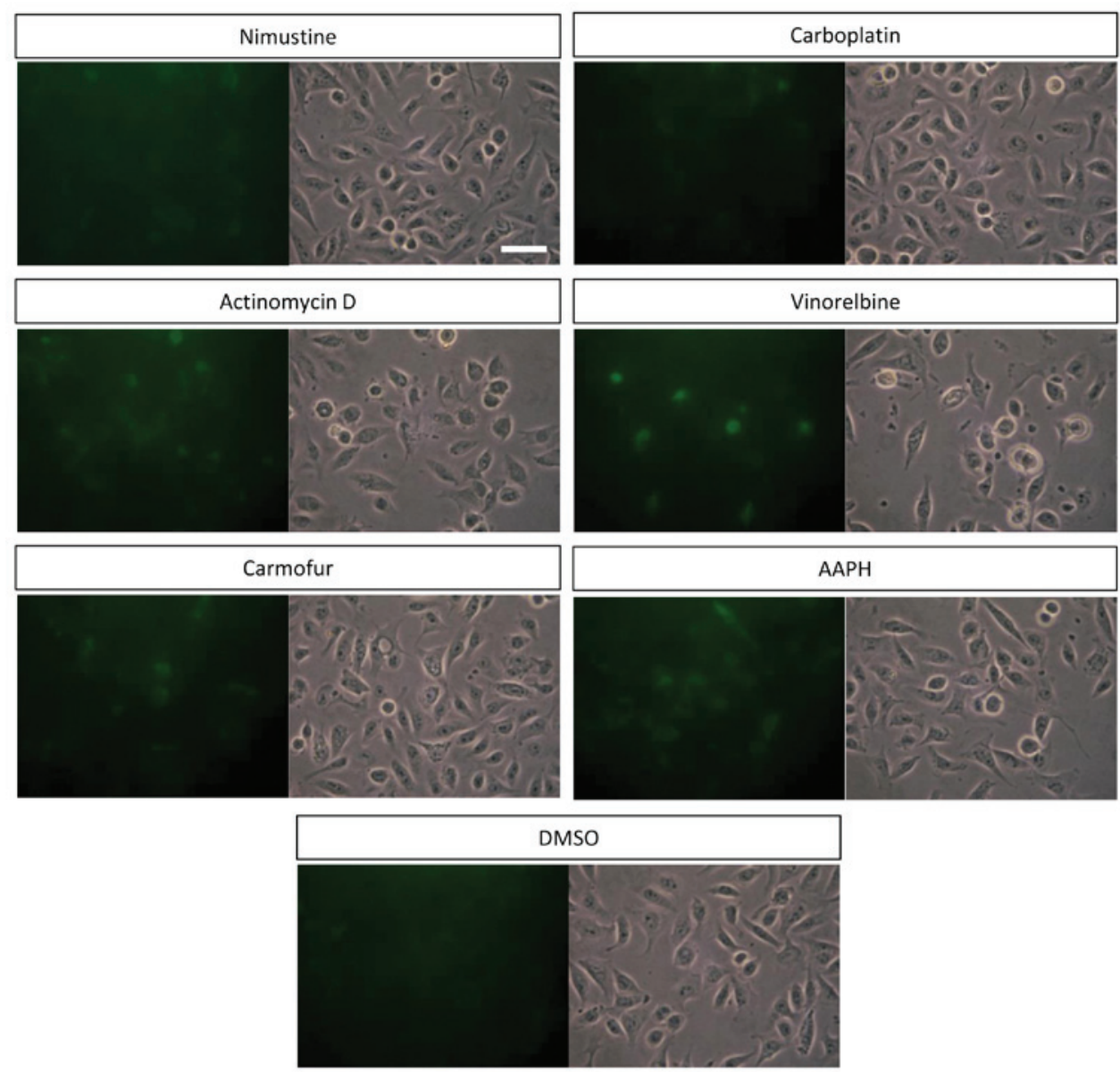

Figure 1. Cell staining of DLD-1 cells treated with anticancer drugs using CellROX ${ }^{\circledR}$ Green reagent. DLD-1 cells were treated with $10 \mu \mathrm{M}$ nimustine, actinomycin D, carmofur, carboplatin and vinorelbine, or $10 \mu \mathrm{M}$ AAPH as a positive control for $24 \mathrm{~h}$. Following incubation, cells were stained with CellROX ${ }^{\circledast}$ Green reagent. Left, CellROX ${ }^{\circledR}$ green fluorescence; right, bright field. Scale bar, $50 \mu \mathrm{m}$. AAPH, 2 2'-azobis (2-amidinopropane) dihydrochloride; DMSO, dimethyl sulfoxide.

assay indicated that nimustine and mitoxantrone reduced the intensity of fluorescence to the same extent as AAPH, whereas vinorelbine reduced the intensity of fluorescence (Table I). However, the reduction in fluorescence intensity of anticancer drugs in the ORAC assay was less extensive, compared with in the ORAC $\left(\mathrm{Cu}^{2+}\right)$ assay.

As the reduction of fluorescence intensity by anticancer drugs in the ORAC $\left(\mathrm{Cu}^{2+}\right)$ assay was attributed to the greater stability of a radical structure of anticancer drugs, the calculation of the radical structural energy of anticancer drugs, including doxorubicin, mitoxantrone, cytarabine, gemcitabine, camptothecin, etoposide, paclitaxel, vinblastine, vinorelbine and AAPH was performed. As the radical structural energy of the most stable structure of each anticancer drug, $\Delta(\mathrm{E}+\mathrm{ZPE})$ of doxorubicin was $80.6 \mathrm{kcal} / \mathrm{mol}$, that of mitoxantrone was $87.9 \mathrm{kcal} / \mathrm{mol}$, that of cytarabine was $83.6 \mathrm{kcal} / \mathrm{mol}$, that of gemcitabine was $89.1 \mathrm{kcal} / \mathrm{mol}$, that of camptothecin was $73.1 \mathrm{kcal} / \mathrm{mol}$, that of etoposide was $76.6 \mathrm{kcal} / \mathrm{mol}$ and that of AAPH is $5.7 \mathrm{kcal} / \mathrm{mol}$. Similarly, $\Delta \mathrm{E}$ of paclitaxel was $88.7 \mathrm{kcal} / \mathrm{mol}$, that of vinblastine was $77.3 \mathrm{kcal} / \mathrm{mol}$ and that of vinorelbine was $77.2 \mathrm{kcal} / \mathrm{mol}$. These results indicated that the anticancer drugs were identified as exhibiting a higher radical structural energy compared with AAPH. Therefore, it was concluded that the results of an ORAC $\left(\mathrm{Cu}^{2+}\right)$ assay was not associated with the radical structural energy of an anticancer drug.

\section{Discussion}

Anticancer drugs often cause a variety of adverse events and induction of ROS has been reported as one of the side effects of anticancer therapies (11-14,27,28). However, the ability of numerous anticancer drugs to induce oxidative stress has not been examined. In the present study, the oxidative stress levels induced by 20 anticancer drugs were investigated using cell staining with CellROX ${ }^{\circledR}$ in vitro and in the absence of cells using the ORAC $\left(\mathrm{Cu}^{2+}\right)$ assay. The data of the current study suggests that certain anticancer drugs have the ability to induce oxidative stress. DLD-1 cells treated with actinomycin D, doxorubicin, camptothecin, paclitaxel, vinblastine or vinorelbine exhibited a strong CellROX ${ }^{\circledR}$ signal; however, it cannot be concluded that the ROS signal was directly derived from treatment with the various anticancer drugs. As numerous cells treated with these anticancer drugs died, the drug-treated DLD-1 cells were stained with Annexin V-Cy3 as a marker of apoptosis for $24 \mathrm{~h}$. Cells that were treated with actinomycin $\mathrm{D}$, doxorubicin, mitomycin $\mathrm{C}$, vinblastine or vinorelbine exhibited a signal with Annexin V-Cy3 (data not shown), suggesting that the ROS signal detected in cells treated with these anticancer drugs is partially dependent on cell death. Taken together, these data suggest that anticancer drugs that are $\mathrm{CellROX}{ }^{\circledR}$-positive/ORAC $\left(\mathrm{Cu}^{2+}\right)$-negative, 
Table I. CellROX ${ }^{\circledR}$ signaling intensity in DLD-1 cells and oxidant activity using the ORAC $\left(\mathrm{Cu}^{2+}\right)$ assay in the absence of cells.

\begin{tabular}{|c|c|c|c|}
\hline $\begin{array}{l}\text { Anticancer } \\
\text { drug }\end{array}$ & $\begin{array}{c}\text { CellROX }^{\circledR} \\
\text { signal }\end{array}$ & $\begin{array}{c}\text { Reduction of } \\
\text { fluorescence } \\
\text { intensity in the } \\
\text { ORAC assay, \% }\end{array}$ & $\begin{array}{l}\text { Reduction of } \\
\text { fluorescence } \\
\text { intensity (\%) } \\
\text { in the ORAC } \\
\left(\mathrm{Cu}^{2+}\right) \text { assay }\end{array}$ \\
\hline Nimustine & - & 58.2 & 42.7 \\
\hline Dacarbazine & - & 98.9 & 99.8 \\
\hline Cyclophosphamide & - & 99.5 & 103.0 \\
\hline Temozolomide & - & 102.4 & 106.0 \\
\hline Mitoxantrone & - & 51.8 & 32.2 \\
\hline Doxorubicin & ++ & 83.5 & 89.2 \\
\hline Actinomycin D & ++ & 88.2 & 92.3 \\
\hline Mitomycin C & + & 102.6 & 98.0 \\
\hline Gemcitabine & - & 93.2 & 44.5 \\
\hline Mercaptopurine & + & 99.4 & 78.4 \\
\hline Carmofur & + & 85.8 & 90.5 \\
\hline Fluorouracil & - & 104.3 & 98.9 \\
\hline Cytarabine & - & 106.1 & 110.4 \\
\hline Carboplatin & - & 104.2 & 94.2 \\
\hline Oxaliplatin & - & 103.3 & 101.6 \\
\hline Vinorelbine & +++ & 33.0 & 19.9 \\
\hline Vinblastine & +++ & 79.3 & 49.1 \\
\hline Camptothecin & ++ & 98.9 & 99.5 \\
\hline Paclitaxel & ++ & 110.3 & 101.1 \\
\hline Etoposide & - & 96.9 & 109.8 \\
\hline AAPH & + & 60.0 & 53.5 \\
\hline DMSO & - & 100.0 & 100.0 \\
\hline
\end{tabular}

-, none or weak; +, slight; ++, moderate; +++, severe. AAPH, 2 2'-azobis (2-amidinopropane) dihydrochloride; DMSO, dimethyl sulfoxide; ORAC $\left(\mathrm{Cu}^{2+}\right)$ assay, oxygen radical absorbance capacity assay in the presence of copper.

such as actinomycin D, doxorubicin, mitomycin C, carmofur, mercaptopurine, camptothecin and paclitaxel disrupt the oxidant-antioxidant balance.

The ORAC and ORAC $\left(\mathrm{Cu}^{2+}\right)$ assays were applied in order to analyze the oxidant activities of anticancer drugs in the absence of cells. In the ORAC assay, the abilities of nimustine and mitoxantrone to reduce the fluorescence intensity were comparable to that of AAPH, whereas vinorelbine markedly reduced the fluorescence intensity. The ORAC $\left(\mathrm{Cu}^{2+}\right)$ assay demonstrated that the reduction in fluorescence intensity of anticancer drugs was enhanced by the addition of $\mathrm{Cu}^{2+}$, such that gemcitabine and vinblastine reduced the fluorescence intensities to an equal extent as nimustine, and mitoxantrone. Although these anticancer drugs were predicted to be able to reduce the fluorescence intensity of fluorescein similar to the radical initiator $\mathrm{AAPH}$, the underlying mechanism remains unknown. Therefore, the radical structural energies of anticancer drugs were estimated using computational chemistry. The radical structural energies of the anticancer drugs were identified to be less stable compared with those of AAPH. This suggests that these anticancer drugs reduced the fluorescence intensity through a different mechanism compared with AAPH.
Furthermore, nimustine, mitoxantrone and gemcitabine were identified as CellROX ${ }^{\circledR}$-negative/ORAC $\left(\mathrm{Cu}^{2+}\right)$-positive. This may be due to an active antioxidant system against nimustine, mitoxantrone and gemcitabine being present in the cells. However, further analyses are required to elucidate a mechanism of action for each anticancer drug.

The signals of CellROX ${ }^{\circledR}$ indicated the presence of intracellular ROS in DLD-1 cells treated with each anticancer drug. Hence, the CellROX ${ }^{\circledR}$-positive anticancer drugs, actinomycin D, doxorubicin, mitomycin $\mathrm{C}$, carmofur, mercaptopurine, camptothecin, paclitaxel, vinblastine, and vinorelbine, ultimately induce oxidative stress in the cells and may cause side effects in cancer patients. Furthermore, the results of the ORAC $\left(\mathrm{Cu}^{2+}\right)$ assay implied that ORAC $\left(\mathrm{Cu}^{2+}\right)$-positive anticancer drugs, nimustine, mitoxantrone, gemcitabine, vinblastine, and vinorelbine, may themselves be oxidants. In addition, the present results suggest that CellROX ${ }^{\circledR}$-positive/ORAC $\left(\mathrm{Cu}^{2+}\right)$ -positive anticancer drugs such as vinblastine and vinorelbine have a strong capacity to induce oxidative stress.

In conclusion, the results of the present study demonstrate that certain anticancer drugs have the ability to induce oxidative stress in the presence or absence of cells. The profiling of anticancer drugs in this study may provide an insight into the ROS-induced mechanisms of anticancer drugs. A detailed examination of the induction of oxidative stress by anticancer drugs may aid in developing novel approaches to reduce the side effects of cancer therapies.

\section{Acknowledgements}

The present study was funded by a Grant-in-Aid for Scientific Research of Seikei University (grant no. 2014).

\section{References}

1. Yoshpe-Purer Y and Eylan E: Disinfection of water by hydrogen peroxide. Health Lab Sci 5: 233-238, 1968.

2. Tauber AI and Babior BM: Evidence for hydroxyl radical production by human neutrophils. J Clin Invest 60: 374-379, 1977.

3. Rosen $\mathrm{H}$ and Klebanoff SJ: Bactericidal activity of a superoxide anion-generating system. A model for the polymorphonuclear leukocyte. J Exp Med 149: 27-39, 1979.

4. Sawyer DT and Tsang PK: The interactions of superoxide ion (O2-.) with metallo-porphyrins [(C1(8)TPP)M, M=Fe, Mn, Co $\mathrm{Zn}$; models for biological systems and superoxide dismutases. Free Radic Res Commun 12-13: 75-86, 1991.

5. Dreher D and Junod AF: Differential effects of superoxide, hydrogen peroxide and hydroxyl radical on intracellular calcium in human endothelial cells. J Cell Physiol 162: 147-153, 1995.

6. Landriscina M, Maddalena F, Laudiero G and Esposito F: Adaptation to oxidative stress, chemoresistance, and cell survival. Antioxid Redox Signal 11: 2701-2716, 2009.

7. Cross CE, Halliwell B, Borish ET, Pryor WA, Ames BN, Saul RL, McCord JM and Harman D: Oxygen radicals and human disease. Ann Intern Med 107: 526-545, 1987.

8. Klaunig JE, Wang Z, Pu X and Zhou S: Oxidative stress and oxidative damage in chemical carcinogenesis. Toxicol Appl Pharmacol 254: 86-99, 2011.

9. Weinberg F and Chandel NS: Reactive oxygen species-dependent signaling regulates cancer. Cell Mol Life Sci 66: 3663-3673, 2009.

10. Trachootham D, Alexandre J and Huang P: Targeting cancer cells by ROS-mediated mechanisms: A radical therapeutic approach? Nat Rev Drug Discov 8: 579-591, 2009.

11. Mercuro G, Cadeddu C, Piras A, Dessì M, Madeddu C, Deidda M, Serpe R, Massa E and Mantovani G: Early epirubicin-induced myocardial dysfunction revealed by serial tissue Doppler echocardiography: Correlation with inflammatory and oxidative stress markers. Oncologist 12: 1124-1133, 2007. 
12. Cadeddu C, Piras A, Mantovani G, Deidda M, Dessì M, Madeddu C, Massa E and Mercuro G: Protective effects of the angiotensin II receptor blocker telmisartan on epirubicin-induced inflammation, oxidative stress, and early ventricular impairment. Am Heart J 160: 487.e1-e7, 2010.

13. Minotti G, Menna P, Salvatorelli E, Cairo G and Gianni L: Anthracyclines: Molecular advances and pharmacologic developments in antitumor activity and cardiotoxicity. Pharmacol Rev 56: 185-229, 2004.

14. Fang J, Nakamura $\mathrm{H}$ and Iyer AK: Tumor-targeted induction of oxystress for cancer therapy. J Drug Target 15: 475-486, 2007.

15. Yamada T,Egashira N,Imuta M, Yano T, Yamauchi Y, Watanabe $H$ and Oishi R: Role of oxidative stress in vinorelbine-induced vascular endothelial cell injury. Free Radic Biol Med 48: 120-127, 2010.

16. DeLoughery Z, Luczak MW and Zhitkovich A: Monitoring $\mathrm{Cr}$ intermediates and reactive oxygen species with fluorescent probes during chromate reduction. Chem Res Toxicol 27: 843-851, 2014.

17. Okamura DM, Bahrami NM, Ren S, Pasichnyk K, Williams JM, Gangoiti JA, Lopez-Guisa JM, Yamaguchi I, Barshop BA, Duffield JS and Eddy AA: Cysteamine modulates oxidative stress and blocks myofibroblast activity in CKD. J Am Soc Nephrol 25: 43-54, 2014.

18. Ting $\mathrm{CH}$, Huang $\mathrm{HN}$, Huang TC, Wu CJ and Chen JY: The mechanisms by which pardaxin, a natural cationic antimicrobial peptide, targets the endoplasmic reticulum and induces c-FOS. Biomaterials 35: 3627-3640, 2014.

19. Cao G, Alessio HM and Cutler RG: Oxygen-radical absorbance capacity assay for antioxidants. Free Radic Biol Med 14: 303-311, 1993.

20. Cao G, Verdon CP, Wu AH, Wang H and Prior RL: Automated assay of oxygen radical absorbance capacity with the COBAS FARA II. Clin Chem 41: 1738-1744, 1995.
21. Ou B, Hampsch-Woodill M and Prior RL: Development and validation of an improved oxygen radical absorbance capacity assay using fluorescein as the fluorescent probe. J Agric Food Chem 49: 4619-4626, 2001.

22. Dávalos A, Gómez-Cordovés C and Bartolomé B: Extending applicability of the oxygen radical absorbance capacity (ORAC-fluorescein) assay. J Agric Food Chem 52: 48-54, 2004.

23. Huang D, Ou B and Prior RL: The chemistry behind antioxidant capacity assays. J Agric Food Chem 53: 1841-1856, 2005.

24. Jomova $\mathrm{K}$ and Valko $\mathrm{M}$ : Advances in metal-induced oxidative stress and human disease. Toxicology 283: 65-87, 2011.

25. Gupte A and Mumper RJ: Elevated copper and oxidative stress in cancer cells as a target for cancer treatment. Cancer Treat Rev 35: 32-46, 2009.

26. Kawashima T, Manda S, Uto Y, Ohkubo K, Hori H, Matsumoto K, Fukuhara K, Ikota N, Fukuzumi S, Ozawa T, et al: Kinetics and mechanism for the scavenging reaction of the 2,2-diphenyl-1-picrylhydrazyl radical by synthetic artepillin $\mathrm{C}$ analogues. Bull Chem Soc Jpn 85: 877-883, 2012.

27. Tewey KM, Rowe TC, Yang L, Halligan BD and Liu LF: Adriamycin-induced DNA damage mediated by mammalian DNA topoisomerase II. Science 226: 466-468, 1984.

28. Vrdoljak AL, Berend S, Zeljezić D, Piljac-Zegarac J, Plestina S, Kuca K, Radić B, Mladinić M and Kopjar N: Irinotecan side effects relieved by the use of HI- 6 oxime: In vivo experimental approach. Basic Clin Pharmacol Toxicol 105: 401-409, 2009. 